\title{
APERIODIC FLUX VARIABILITY IN A $0535+262$
}

\author{
MARK H. FINGER \\ Compton Observatory Science Support Center/USRA \\ Goddard Space Flight Center \\ ROBERT B. WILSON AND B. ALAN HARMON \\ NASA/Marshall Space Flight Center \\ AND \\ WILLIAM S. PACIESAS \\ University of Alabama Huntsville
}

\begin{abstract}
A "giant" outburst of A 0535+262, a transient X-ray binary pulsar, was observed in 1994 February and March with the Burst and Transient Source Experiment (BATSE) onboard the Compton Gamma-Ray Observatory. During the outburst power spectra of the hard X-ray flux contained a QPO-like component with a FWHM of approximately $50 \%$ of its center frequency. Over the course of the outburst the center frequency rose smoothly from $35 \mathrm{mHz}$ to $70 \mathrm{mHz}$ and then fell to below $40 \mathrm{mHz}$. We compare this QPO frequency with the neutron star spin-up rate, and discuss the observed correlation in terms of the beat frequency and Keplerian frequency QPO models in conjunction with the Ghosh-Lamb accretion torque model.
\end{abstract}

\section{Introduction}

A $0535+262$ is a $103 \mathrm{~s} \mathrm{X}$-ray pulsar in a binary system with the Be star HDE 245770. Since its initial discovery in 1975 (Rosenberg et al. 1975), the source has been frequently observed to undergo transient outbursts. The outbursts show a range of peak intensities, with the largest reaching $3 \mathrm{Crab}$ in the $2-10 \mathrm{keV}$ band. For a review of previous observations see Giovannelli \& Graziati (1992).

A major outburst of A $0535+262$ occurred in 1994 February and March (Wilson et al. 1994a). Hard X-ray observations with BATSE were made continuously during the $\mathbf{5 0}$ day duration of the outburst. At the peak of 
the outburst the intrinsic spin up rate determined from pulse timing was approximately $1.210^{-11} \mathrm{~Hz} \mathrm{~s}^{-1}$ (Wilson et al. 1994b), clearly indicating the presence of an accretion disk. The formation of a transient accretion disk during "giant" outbursts has previously been inferred from optical and UV observations (Motch et al. 1991).

During 27 days of the outburst a broad quasi-periodic oscillation (QPO) like feature appeared in Fourier power spectra of the flux (Finger et al. 1994b). One possible explanation for this feature is the beat frequency model (Alpar \& Shaham 1985; Lamb et al. 1985). In this model blobs of matter, in the process of being entrained in the neutron star's magnetic field, orbit the neutron star at approximately the Keplerian frequency of the inner edge of the accretion disk, accreting at a rate that is modulated by the magnetic field. This produces a peak in the power spectra at the beat frequency between the Keplerian and the pulsar spin frequencies. Another possible model is that the inner edge of the accretion disk contains structures that persist for a few cycles around the neutron star, and modulate the observed flux by obscuration (Van der Klis et al. 1987). In this case, the power spectral feature should be located near the Keplerian frequency of the disk inner edge.

Both of these models predict a simple relationship between the QPO frequency and the rate of mass accretion through the disk. The accretion rate also determines the torque on the neutron star. We compare the observed relationship between the neutron star spin up rate and the QPO frequency with the predictions of the beat frequency or Keplerian frequency models combined with the Ghosh \& Lamb (1979) accretion torque model. The beat frequency model does best at predicting the trend of the data, but the Keplerian frequency model is not ruled out by the observations.

\section{Observations and Analysis}

BATSE (Fishman et al. 1989) has eight unshielded planar scintillation detectors oriented in the corner directions of a cube. Its full sky field of view allows the continuous monitoring of transient outbursts.

Pulsed hard X-ray emission from A $0535+262$ was first detected with BATSE on January 28th (TJD 9380). The flux initially remained at a low level, but then on February 3 the flux began to rise quickly (Finger et al. 1994a), peaking on February 18 (Wilson et al. 1994a,b). The last BATSE pulsed flux detection was on March 20, fifty days after the first detection. The light curve of the February-March outburst as determined by the Earth occultation method is shown in panel A of Fig. 1.

A pulse timing analysis was performed to determine the intrinsic spinup rate of $A 0535+262$. This analysis used the binary orbital parameters 

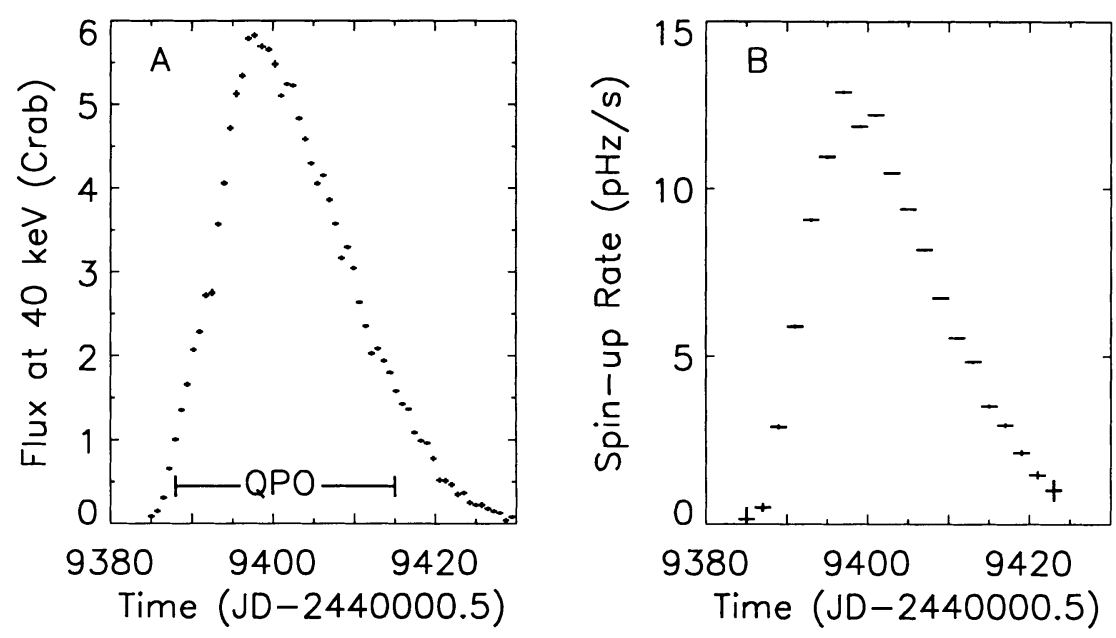

Figure 1. A) shows the A $0535+262$ flux history determined from Earth occultations. Also shown is the interval of QPO detections. B) shows the intrinsic spin-up of A $0535+262$ during the outburst.

recently determined by Finger et al. (1993), based on a series of weak outbursts of A $0535+262$ that occurred near periastron passage in the three orbits previous to the February-March outburst. The spin-up rate during the outburst is shown in panel B of Fig. 1.

Aperiodic variability in the source flux was noticed early in the outburst (Finger et al. 1994b). Daily average power spectra were made from the DISCLA channel 1 rates $(20-50 \mathrm{keV}, 1.024 \mathrm{~s}$ resolution) after subtraction of the daily mean pulse profile. The power spectrum for February 19 is shown in panel A of Fig. 2. These power spectra consist of an approximately $1 / f$ power-law component extending from at least $5 \mathrm{mHz}$ to $0.5 \mathrm{~Hz}$, and a significant concentration of noise power in a bump centered in the 35$70 \mathrm{mHz}$ range. For convenience we will call this bump in the power spectra a QPO, although the bump typically has a FWHM/center frequency of $50 \%$ or slightly greater, and therefore does not strictly meet the QPO definition used in connection with LMXBs. Typical fractional r.m.s. amplitudes of the coherent, power-law $(10-500 \mathrm{mHz})$, and QPO components were $20 \%$, $15 \%$, and $9 \%$, respectively. The QPO was detectable for an interval of 27 days indicated in panel A of Fig. 1. Panel B of Fig. 2 shows the history of the QPO center frequency, obtained by fitting the daily power spectra with the sum of Gaussian and power-law models. As the outburst progressed the center frequency rose smoothly from $35 \mathrm{mHz}$ to $70 \mathrm{mHz}$ near the peak of the outburst, and returned slowly to $35 \mathrm{mHz}$. 

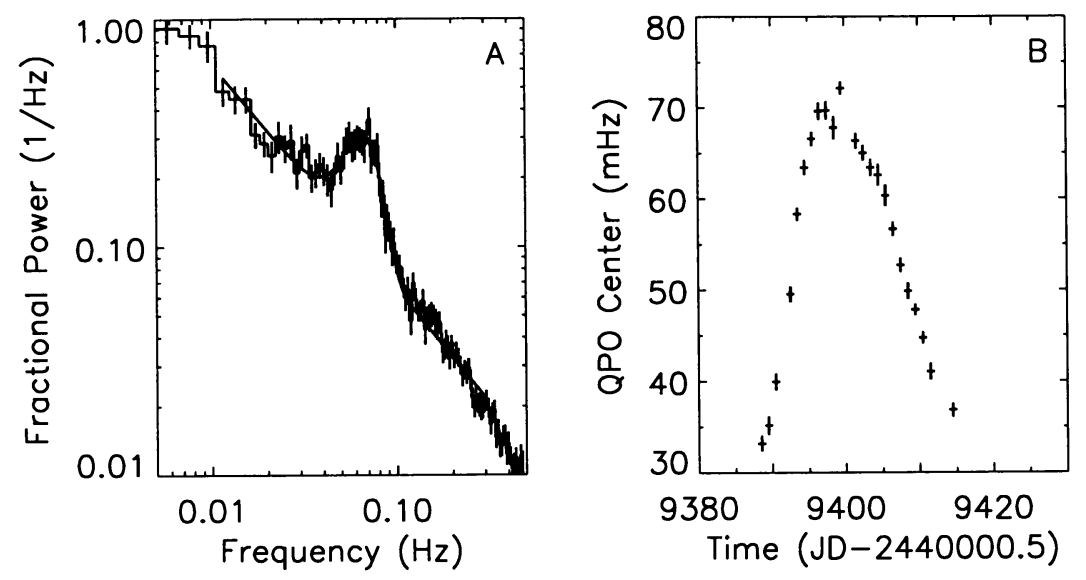

Figure 2. A) shows the mean power spectrum for February 19. The best fit model is superposed within the fit interval. B) shows the center frequency of the Gaussian QPO component of the power spectral model.

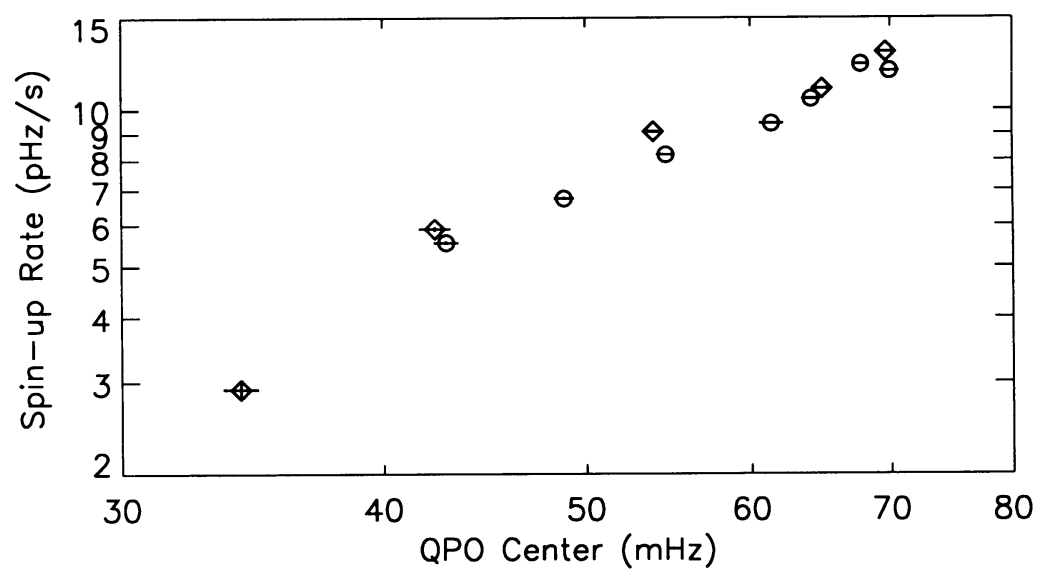

Figure 3. Comparison of intrinsic spin-up rate of A $0535+262$ during the outburst and the QPO center frequency. Diamond symbols are used during the rise of the outburst and circles during the decline.

Fig. 3 compares the QPO center frequency and the neutron star spin up rate. For the plot, pairs of center frequencies were interpolated to the midpoint of the spin up rate measurements. The QPO frequency and the spin up rate are seen to be highly correlated. The tracks from the rise and the fall of the outburst are amazingly close, with spin up on the rise being only slightly higher than spin up on the fall at the same QPO frequency. 


\section{Discussion}

Simple theoretical predictions of the relationship between the QPO center frequency and the spin up rate can be obtained by combining either the beat frequency model, or the Keplerian frequency model with the Ghosh \& Lamb (1979) accretion torque model. The Ghosh \& Lamb model gives the accretion torque as

$$
N=\dot{M} \sqrt{G M r_{0}} n\left(\omega_{\mathrm{s}}\right)
$$

where $\dot{M}$ is the accretion rate, $M$ is the neutron star mass, and $r_{0}$ is the radius of the inner edge of the accretion disk. The dimensionless torque function $n\left(\omega_{\mathrm{s}}\right)$ depends only on the fastness parameter $\omega_{s}$ which is the ratio of the neutron star spin frequency $\nu_{\text {ns }}$ to the Keplerian frequency at the inner edge of the accretion disc $\nu_{\mathrm{K}}$. The radius of the inner edge of the accretion disk is given by

$$
r_{0}=(G M)^{1 / 3}\left(2 \pi \nu_{\mathrm{K}}\right)^{-2 / 3}=\eta \mu^{4 / 7}(2 G M)^{-1 / 7} \dot{M}^{-2 / 7}
$$

where $\eta$ is a geometry-dependent constant that Ghosh \& Lamb computed to be 0.52 , and $\mu$ is the neutron star magnetic moment. If we assume that the torque $N$ acts on the solid body moment of inertia of $2 / 5 M R^{2}$, the spin-up rate may be written as

$$
\dot{\nu}_{\mathrm{ns}}=\aleph n\left(\omega_{s}\right) \nu_{\mathrm{K}}^{2} \text { where } \aleph=\frac{5 \pi \eta^{3.5} \mu^{2}}{\sqrt{2} G M^{2} R^{2}} .
$$

The dimensionless constant $\aleph$ has a value of $2.110^{-9}$ for the representative values of $\eta=1, \mu=10^{31} \mathrm{G} \mathrm{cm}^{3}, M=1.4 \mathrm{M}_{\odot}$, and $R=10^{6} \mathrm{~cm}$.

A plot of $\dot{\nu}_{\text {ns }} / \nu_{\mathrm{K}}^{2}$ versus the fastness parameter $\omega_{\mathrm{s}}$ will therefore yield a measurement of $\aleph n\left(\omega_{\mathrm{s}}\right)$. This is shown in Fig. 4 for both the beat frequency and Keplerian frequency models. Also shown on the plots is the theoretical relationship for several values of $\aleph$ using the approximation for $n\left(\omega_{\mathrm{s}}\right)$ given by Ghosh \& Lamb (1979). For both the beat frequency model and the Keplerian frequency model the approximate scaling of $\dot{\nu}_{\mathrm{ns}} \propto \nu_{\mathrm{K}}^{2}$ is born out by the data. For the beat frequency model, the observations are consistent with $\aleph n\left(\omega_{\mathrm{s}}\right)$ being a slowly varying function that decreases with increasing $\omega_{\text {s }}$ (decreasing $\left.\dot{M}\right)$, although detailed agreement between observation and model prediction is not achieved. For the Keplerian frequency model, the observed form of $\aleph n\left(\omega_{\mathrm{s}}\right)$ rises at low $\omega_{\mathrm{s}}$ and then falls at higher $\omega_{\mathrm{s}}$. The agreement between observations and model prediction is worse than in the beat frequency model case. The observations therefore favour the beat frequency model, for which we estimate a value of $\aleph=(2.5 \pm 0.5) 10^{-9}$.

For assumed values of $\eta=1, R=10^{6} \mathrm{~cm}$, and $M=1.4 \mathrm{M}_{\odot}$, we compute a polar magnetic field of $B=2 \mu / R^{3}=210^{13} \mathrm{G}$. Observations with 

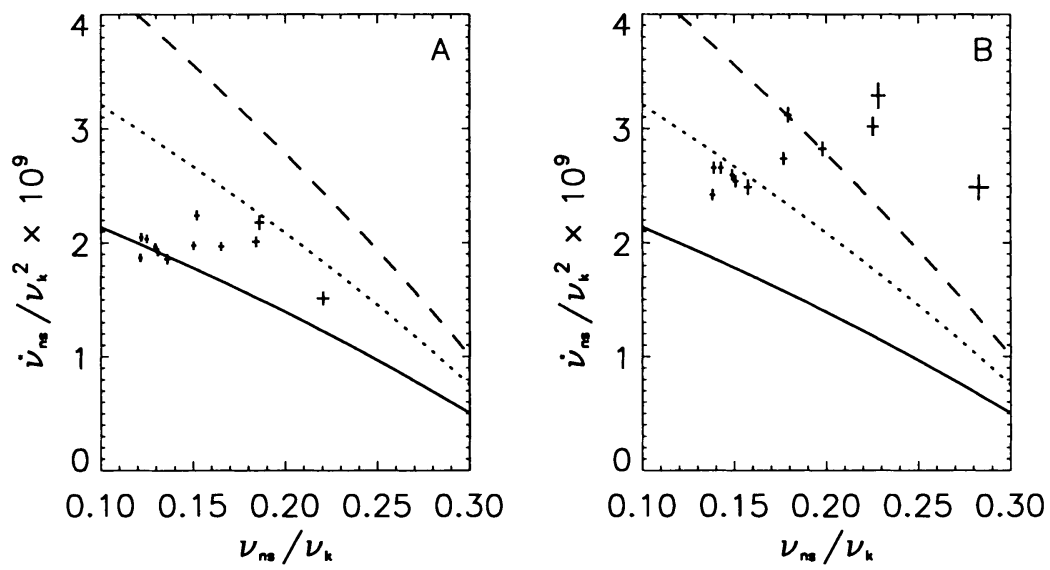

Figure 4. The ratio of the neutron star spin-up rate to the Keplerian frequency squared for the beat frequency (panel A) and Keplerian frequency (panel B) models. The curves give the Ghosh-Lamb prediction, for values of the dimensionless constant $\aleph$ of $2.010^{-9}$ (solid), $3.010^{-9}$ (dotted), and $4.010^{-9}$ (dashed).

OSSE during this same outburst of A $0535+262$ revealed a cyclotron line at an energy of $110 \mathrm{keV}$ (Grove et al. 1994), or a polar magnetic field of $B=9.510^{12} \mathrm{G}$. Given the number of poorly known parameters, this rough agreement is encouraging.

Acknowledgements. M.H.F. would like to thank J. van Paradijs, M. van der Klis, and L. Bildsten for useful discussions.

\section{References}

Alpar, M.A. \& Shaham, J. 1985, Nat 316, 239

Finger, M.H. et al. 1993, in Evolution of X-ray Binaries, S.S. Holt \& C.S. Day (Eds.), AIP Conf. Proc. Vol. 408, p. 459

Finger, M.H., Wilson, R.B. \& Hagedon, K.S. 1994a, IAU Circ. 5931

Finger, M.H., Wilson, R.B. \& Harmon, B.A. 1994b, IAU Circ. 5934

Fishman, G.J. et al. 1989, Proceedings GRO Science Workshop, GSFC, p. 2-39

Ghosh, P. \& Lamb, F.K. 1979, ApJ 234, 296

Giovannelli, F. \& Graziati, L.S. 1992, Space Sci. Rev. 59, 1

Grove, E. et al. 1994, ApJ (in press)

Lamb, F.K. et al. 1985, Nat 317, 681

Motch, C. et al. 1991, ApJ 369, 490

Rosenberg, F.D. et al. 1975, Nat 256, 628

Van der Klis, M. et al. 1987, ApJ 313, L19

Wilson, R.B., Harmon, B.A. \& Finger, M.H. 1994a, IAU Circ. 5933

Wilson, R.B. et al. 1994b, IAU Circ. 5945 


\section{Discussion}

P. Ghosh: Your determination of $\frac{d \ln \nu_{k}}{d \ln M}$ (which is nominally 2/7, as verified by early QPO work on $\mathrm{LMXB}$ ) is very likely going to be the best measurement so far. This has very important implications for the state of the inner accretion disk in X-ray pulsars. The value is in agreement with the usual one-temperature and gas-pressure dominated disk, and not with some other dark models.

A. Alpar: The observation of the beat frequency does not depend on the existence of beaming or polar caps if the field is weak enough, as in LMXBs.

M. Finger: This is a HMXB with beaming and the observation of $\nu_{\text {rotation }}$ is expected.

A. Alpar: To see the inclination dependence comparatively and to

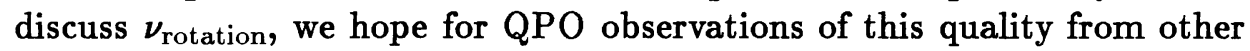
HMXBs.

J. van Paradijs: This is a response to the remark by P. Ghosh. One can derive relations between $\dot{\nu}$ and $\nu_{\mathrm{K}}$ for different disk models. It turns out that the only disk model (from a sample of models published by P. Ghosh) that fits the approximately quadratic dependence of $\dot{\nu}$ on $\nu_{\mathrm{K}}$ is that of a classical gas-pressure dominated disk; a radiation-pressure dominated disk, or a twotemperature disk, lead to very different $\dot{\nu}\left(\nu_{\mathrm{K}}\right)$ relations, inconsistent with the one observed for A $0535+262$. 